\title{
Finite temperature vacancy formation thermodynamics: local harmonic and quasiharmonic studies
}

\author{
L Zhaoł, R Najafabadił and D J Srolovitz†£ \\ $\dagger$ Applied Physics Program, University of Michigan, Ann Arbor, MI 48109, USA \\ $\ddagger$ Department of Materials Science and Engineering, University of Michigan, Ann Arbor, MI \\ 48109, USA
}

Received 1 September 1992, accepted for publication 16 November 1992

\begin{abstract}
The vacancy formation thermodynamics in six FCC metals $\mathrm{Ag}, \mathrm{Au}, \mathrm{Cu}, \mathrm{Ni}, \mathrm{Pd}$ and $\mathrm{Pt}$ are determined from atomistic simulations as a function of temperature. This investigation is performed using the embedded atom method interatomic potentials and the finite temperature properties are determined within the local harmonic and the quasiharmonic frameworks. The temperature dependence of the vacancy formation free energy, entropy, enthalpy and vacancy formation volume are determined. We find that the temperature dependence of the vacancy formation energy can make a significant contribution to the vacancy concentration at high temperatures. An additional goal of the present study is to evaluate the accuracy of the local harmonic method under circumstances in which the excess entropy associated with the formation of a defect is very small. Our data demonstrate that while the errors associated with determining the vacancy formation entropy in the local harmonic model are large, a simple extension to the local harmonic method yields thermodynamic properties comparable to that obtained in the quasiharmonic model, but with much higher computational efficiency.
\end{abstract}

\section{Introduction}

In many materials, the presence and motion of vacancies dictate diffusivities, contribute to electrical resistivity and carrier recombination, and provide the mechanism for void growth during high-temperature deformation. Therefore, knowledge of vacancy thermodynamics is an essential ingredient in understanding a wide range of physical properties. All thermodynamic functions associated with the presence of vacancies may be derived from knowledge of the vacancy formation free energy. Therefore, it is not surprising that vacancy formation energies in metals have received considerable theoretical and experimental attention.

Recently, Gillan [1] has studied vacancy formation energies by applying the pseudopotential method and local-density approximation for exchange and correlation in a periodically repeating supercell geometry. While this is a first-principle approach, it is limited to zero Kelvin. Finite-temperature studies, based upon empirical descriptions of atomic bonding and the molecular dynamics [2] and Monte Carlo $[3,4]$ methods, have been able to overcome these limitations. In the present study, we employ embedded atom method (EAM) potentials [5] to describe atomic interactions, classical lattice dynamics methods and a novel finite temperature simulation approach to examine vacancy formation thermodynamics in six face centred cubic (FCC) metals as a function of temperature.

§ e-mail: srol@dodo.engin.umich.edu 
A variety of experimental methods have been used to determine vacancy formation energies [6]. These include the measurement of electrical resistivity of quenched and irradiated metals, high-temperature measurement of heat capacity and thermal expansion, comparison of dilatomic and $\mathrm{x}$-ray measurements of thermal expansion, determination of stored energy release of irradiated and cold-worked specimens, diffusion measurements, and internal friction studies. These various methods yield roughly comparable results. In most materials, a wide variety of point defects and more extended defects such as dislocations and grain boundaries exist. The difficulties in sorting out the effects of these different defects and their interactions in the experimental measurements is inherent, such that some uncertainty in the interpretation of the experimental results remains. In spite of these difficulties, experimental vacancy formation energy data have been used in the construction of many interatomic potentials, including the EAM potentials [5].

The vacancy concentration in a solid is simply related to the vacancy formation free energy $G_{\mathrm{v}}$ by

$$
N_{\mathrm{v}} /\left(N+N_{\mathrm{v}}\right)=\exp \left(-G_{\mathrm{v}} / k T\right)
$$

where $N_{\mathrm{v}}$ and $N$ represent the number of vacancies and atoms in the crystal, respectively, and $k T$ is the thermal energy. Equation (1) can be rewritten as:

$$
N_{\mathrm{v}} / N=\exp \left(S_{\mathrm{v}} / k\right) \exp \left(-U_{\mathrm{v}} / k T\right) \exp \left(-P V_{\mathrm{v}} / k T\right)
$$

where we have simply rewritten the vacancy formation free energy $G_{\mathrm{v}}$ in terms of its components: the entropy $S_{\mathrm{v}}$, internal energy $U_{\mathrm{v}}$ and the work term $P V_{\mathrm{v}}$ associated with vacancy formation, where $V_{v}$ is the vacancy formation volume. Equation (2) also makes use of the fact that the vacancy concentration is typically much smaller than the concentration of atoms, $N_{\mathrm{v}} \ll N$. It is this form of (1) that is commonly employed in the experimental determination of $U_{\mathrm{v}}$ and $V_{\mathrm{v}} . U_{\mathrm{v}}$ is obtained by measuring a quantity that is proportional to the vacancy concentration as a function of temperature at constant pressure and plotting the data in an Arrhenius form, $\ln \left(N_{\mathrm{v}}\right)$ versus $(1 / T)$, at zero pressure. $V_{\mathrm{v}}$ is obtained from the slope of a plot of $\ln N_{\mathrm{v}}$ versus $P$ at constant temperature. The application of these methods is simplest when the vacancy formation energy is independent of temperature and pressure. The question of the magnitude of the variation of the vacancy formation free energy with temperature and pressure is therefore of considerable importance. It has been argued [7] that for most monatomic crystals, the entropy, energy, and vacancy formation volume are nearly constant.

The focus of the present work is the application of a free energy simulation method to calculate the equilibrium structure and thermodynamics of vacancy-containing crystals. In particular, the present paper examines an otherwise perfect crystal containing a single vacancy. We determine the vacancy formation energy and volume as a function of temperature for six FCC metals: $\mathrm{Ag}, \mathrm{Au}, \mathrm{Cu}, \mathrm{Ni}, \mathrm{Pd}$ and Pt. Our results confirm the validity of treating the vacancy formation energy and volume as constants at intermediate temperatures. Although the temperature dependence of the vacancy formation free energy is not large, ignoring it will yield significant errors in the vacancy concentration at elevated temperatures. In addition to studying the thermodynamics of vacancy formation in crystalline metals, the present study serves as a comparison between the approximate free energy simulation method and the more rigorous quasiharmonic model. Based upon this comparison, we propose simple extensions to the free energy simulation method that combine the accuracy of the quasiharmonic model with the efficiency of the current free energy simulation method. 
The free energy simulation method is based upon several simple approximations. The central assumption is that there are no correlations between the vibrations of one atom and the other atoms, i.e. the terms in the dynamical matrix that couple one atom to another are assumed to be zero. This is equivalent to treating each atom in the system as an individual Einstein oscillator. Since each atom vibrates in its own, individual quadratic potential well, this approximation to the free energy is known as the local harmonic (LH) model [8]. The other important assumption employed in the present study involves the description of atomic interactions. All of the simulation results reported in the present work were obtained using the empirical EAM potentials [5]. The EAM potentials are also used to describe the shape of the potential wells in which the atoms vibrate. The free energy minimization method, as its name suggests, is based upon minimizing the LH free energy functional with respect to the positions of all of the atoms in order to determine the equilibrium atomic structure at arbitrary temperature and pressure. Once the atomic structure is known, the free energy and all other thermodynamic quantities may be directly determined.

The free energy minimization method is both physically and mathematically simple and is much more computationally efficient than any other method for determining the free energy of a defected solid which is consistent with its atomic structure. The central approximation of the LH model (i.e. each atom is an independent vibrator) reduces the calculation of the vibrational contributions to the free energy from one of diagonalizing a $3 N \times 3 N$ dynamical matrix (e.g. in the quasiharmonic method $[9,10]$ ) to the calculation of $N$ determinants of $3 \times 3$ matrices in an $N$-atom system. This results in a reduction in computational work by at least a factor of $N$ and more typically by $N^{2}$. Since atomistic simulations typically involve between hundreds and millions of atoms, this is a considerable efficiency improvement.

Another advantage of the free energy simulation approach is that it is a selfconsistent treatment of a defect system, i.e. the finite temperature atomic structure and the thermodynamics of that structure are determined self-consistently. By comparison, methods such as the quasiharmonic method are usually applied to atomic structures determined using some other method (e.g. experiment, molecular dynamics or Monte Carlo). In a perfect crystal with a primitive unit cell, the temperature variation of the atomic structure is generally limited to the thermal expansion (unless a phase transition occurs). While the quasiharmonic lattice theory was previously employed to calculate vacancy free energies of formation [11], the atomic structure in the vicinity of the vacancy was not determined self-consistently.

Simulation methods other than the LH method have been employed or could be applied to the determination of the finite temperature thermodynamic properties of defects in solids. While Monte Carlo methods may be combined with thermodynamic integration procedures to accurately predict thermodynamic properties, this approach requires substantial computational power to perform systematic studies. For example, a single step in the integration procedure would require of order one hour on a state-ofthe-art workstation. Therefore, since the determination of thermodynamic properties at large fractions of the melting point would likely require in excess of ten integration steps, the Monte Carlo/thermodynamic integration procedure is very computer time intensive. A cumulant analysis of the internal energy and the enthalpy distribution functions obtained from a Monte Carlo simulation at a single temperature $[10,12]$ requires the same magnitude of computer time as one step in an integration study. With the LH method, on the other hand, determination of the free energy over a wide range of temperatures requires only several seconds on a similar workstation. Unfortunately, this enhanced computational performance comes at the cost of more severe approximations. While 
the Monte Carlo/thermodynamic integration approach is essentially exact, methods based upon the harmonic approximation ignore many potentially important anharmonic effects. Nonetheless, the harmonic approximation is known to yield accurate perfect-crystal free energies at temperatures up to three quarters of the melting temperature [13]. The LH method represents an even more severe approximation than the harmonic approximation itself; i.e. it ignores most of the off diagonal terms in the dynamical matrix. The LH method has been shown to be in excellent agreement with the more exact free energy methods in several applications $[8,14]$. Below, we will show that a simple extension to the LH model can further improve the accuracy of the LH method.

In the present paper, we report vacancy formation free energies and volumes for a series of FCC metals over a wide temperature range $(0 \mathrm{~K}$ to $1000 \mathrm{~K})$. The results obtained from the LH approximation are compared with those obtained from the quasiharmonic $(\mathrm{QH})$ approximation. We expect that the agreement between the $\mathrm{LH}$ and $\mathrm{QH}$ predictions of the vacancy formation entropy will not be as good as for perfect crystals and other types of crystalline defects due to the very small vacancy formation entropy contribution to the vacancy free energy of formation. We employ the present results in order to test extensions to the LH model, obtained by reducing the severity of the approximations inherent to the LH approximation. This extended local harmonic (ELH) is shown to predict thermodynamic properties that are in good agreement with the $\mathrm{QH}$ method over a large temperature range without sacrificing the computational efficiency that has made the $\mathrm{LH}$ model attractive.

The remainder of this paper is organized as follows. In section 2, we briefly outline both the $\mathrm{LH}$ and $\mathrm{QH}$ methods. Vacancy formation thermodynamics results obtained with these methods are presented in section 3. A comparison of the vacancy formation thermodynamics obtained using these two methods is presented after that. Based upon these results, we develop the ELH model and apply it to vacancy formation thermodynamics (section 4).

\section{The local harmonic and quasiharmonic models}

A detailed description of the QH and LH models may be found in [9] and in [8] and [15], respectively. In any harmonic description of the vibrational contributions to the free energy, the first step is to construct the dynamical matrix D

$$
\mathbf{D}_{i \alpha, j \beta}=\frac{\partial^{2} U}{\partial r_{i}^{\alpha} \partial r_{j}^{\beta}}
$$

where $U$ is the static lattice energy, and $r_{i}^{\alpha}$ is the $\alpha$ th Cartesian component of the displacement vector of atom $i$. In general, each of the $(3 N)^{2}$ terms of the dynamical matrix is non-zero. The vibrational density of states $N(\omega)$ of the system is simply the probability distribution of the eigenfrequencies of the system. The eigenfrequencies are determined in terms of the eigenvalues or force constants of the dynamical matrix $k_{i \alpha}$ as $\omega_{i \alpha}=\left(k_{i \alpha} / m_{i}\right)^{1 / 2}$, where $m_{i}$ is the mass of atom $i$. In the $\mathrm{QH}$ approximation, the Helmholtz free energy $A$ is given by

$$
\begin{array}{r}
A=U+k T \int_{0}^{\infty} N(\omega) \ln \left[2 \sinh \left(\frac{\hbar \omega}{2 k T}\right)\right] \mathrm{d} \omega \\
=U+k T \sum_{k=1}^{3 N} \ln \left[2 \sinh \left(\frac{\hbar \omega_{k}}{2 k T}\right)\right]
\end{array}
$$


where the sum is over all $3 N$ eigenfrequencies of the dynamical matrix. As mentioned above, we describe $U$ in the free energy equation and in the determination of the elements of the dynamical matrix in terms of EAM potentials.

In the LH approximation, the vibration of each atom is assumed to be uncorrelated with the vibrations of the other atoms in the system. Therefore, the appropriate eigenfrequencies for atom $i$ are those found by diagonalizing the $3 \times 3$ dynamical matrix of the form $D_{i \alpha, i \beta}$. In the classical limit of the LH model, the free energy is given by

$$
A=U+k T \sum_{i=1}^{N} \sum_{\beta=1}^{3} \ln \left[\frac{\hbar \omega_{i \beta}}{k T}\right]=U+k T \sum_{i=1}^{N} \ln \left[\frac{\hbar|D|^{1 / 6}}{k T \sqrt{m}}\right]
$$

where the summation on $\beta$ is over the three eigenfrequencies of the local dynamical matrix associated with atom $i$. The second equality in (5) indicates that it is not necessary to determine the eigenvalues of the dynamical matrix but, rather, it is sufficient to calculate its determinant $|D|$. It has previously been shown $[8,15]$ that the approximate LH model leads to errors in the free energy of perfect close-packed metal crystals of the order of $1 \%$ at the melting temperature and much less at lower temperatures.

As the name suggests, in the free energy minimization method the equilibrium atomic structure and corresponding thermodynamic properties of a system are determined by minimizing the total $\mathrm{LH}$ free energy of the system (at any temperature and pressure) with respect to the atomic coordinates. For a perfect cubic crystal, the free energy minimization is reduced to a minimization with respect to one variable, the lattice constant, $a_{0}$. In order to obtain an accurate comparison between the $\mathrm{LH}$ and $\mathrm{QH}$ methods, the atomic structures for the QH determination of the vacancy formation energy are assumed to be identical to those determined from the LH free energy minimization. However, the overall volume is relaxed using the $\mathrm{QH}$ model to guarantee that the pressure is zero. While this does not lead to truly self-consistent $\mathrm{QH}$ results, this is a better approximation than is typically employed for determining the atomic structure in $\mathrm{QH}$ calculations.

We insert a vacancy into the crystal by removing one of the atoms from a perfect FCC crystal lattice. The size of the cubic simulation cell is discussed below. Periodic boundary conditions are employed in all three orthogonal directions. The relaxed atomic configuration was determined using a conjugate gradient minimization method which was terminated when the force on each atom was less than $10^{-4} \mathrm{eV} \AA^{-1}$.

The excess vacancy thermodynamic properties are determined as the difference between the property determined in a system containing one vacancy and that in a perfect crystal containing the same number of atoms; i.e. the free energy of the perfect crystal was determined by calculating the free energy per atom in a perfect crystal and multiplying by the number of atoms in the crystal with a vacancy. The vacancy formation free energy is denoted as $G_{\mathrm{v}}=G_{0}-G_{\mathrm{p}}$, where $G_{0}$ is the free energy of the system with a vacancy and $G_{\mathrm{p}}$, is the free energy for the perfect crystal with the same number of atoms. Similarly, the vacancy formation volume is defined as $V_{\mathrm{v}}=V_{0}-V_{\mathrm{p}}$, where $V_{0}$ and $V_{\mathrm{p}}$ are the volumes of systems with a single vacancy and a perfect crystal containing the same number of atoms at the same pressure. The excess vacancy formation entropy and enthalpy are determined from the following expressions: $S_{\mathrm{v}}=-\partial G_{\mathrm{v}} / \partial T$ and $H_{\mathrm{v}}=G_{\mathrm{v}}+T S_{\mathrm{v}}$.

\section{Results and discussion}

\subsection{Perfect-crystal $L H$ and $Q H$ thermodynamics}

The thermodynamic properties of perfect $\mathrm{Ag}, \mathrm{Au}, \mathrm{Cu}, \mathrm{Ni}, \mathrm{Pd}$ and Pt crystals described by 
the LH model and the EAM potentials were determined as a function of temperature at zero pressure. The free energy, entropy and enthalpy of $\mathrm{Cu}$ and $\mathrm{Pd}$ perfect crystals are shown in figures $1(a)-(c)$. For comparison, these figures also show experimental determinations of these quantities [16], where all of the data have been normalized to room temperature, $298.15 \mathrm{~K}$. The agreement between the $\mathrm{LH}$ free energy minimization data and the experimental results for each element over the entire temperature range $(0$ to $1000 \mathrm{~K})$ is excellent. A comparison between perfect-crystal gold free energies determined using the LH model and accurate Monte Carlo results [17] using the same interatomic potential may be found in [15]. Overall, the agreement between the Monte Carlo, experimental, and LH data is excellent. This suggests that the LH model is a good approximation to the true free energy and that the EAM potential is a reasonable representation of the atomic interactions, at least in perfect crystals of gold. We find essentially the same, excellent agreement between experiment and LH data for the other metals described by EAM potentials. Comparison between the LH perfect-crystal free energies and those obtained using the $\mathrm{OH}$ model are also in excellent agreement. For example, we find that the error between the $\mathrm{LH}$ and $\mathrm{QH}$ free energies is approximately $0.14 \%$ at $200 \mathrm{~K}$ and $0.57 \%$ at $1000 \mathrm{~K}$ in gold.
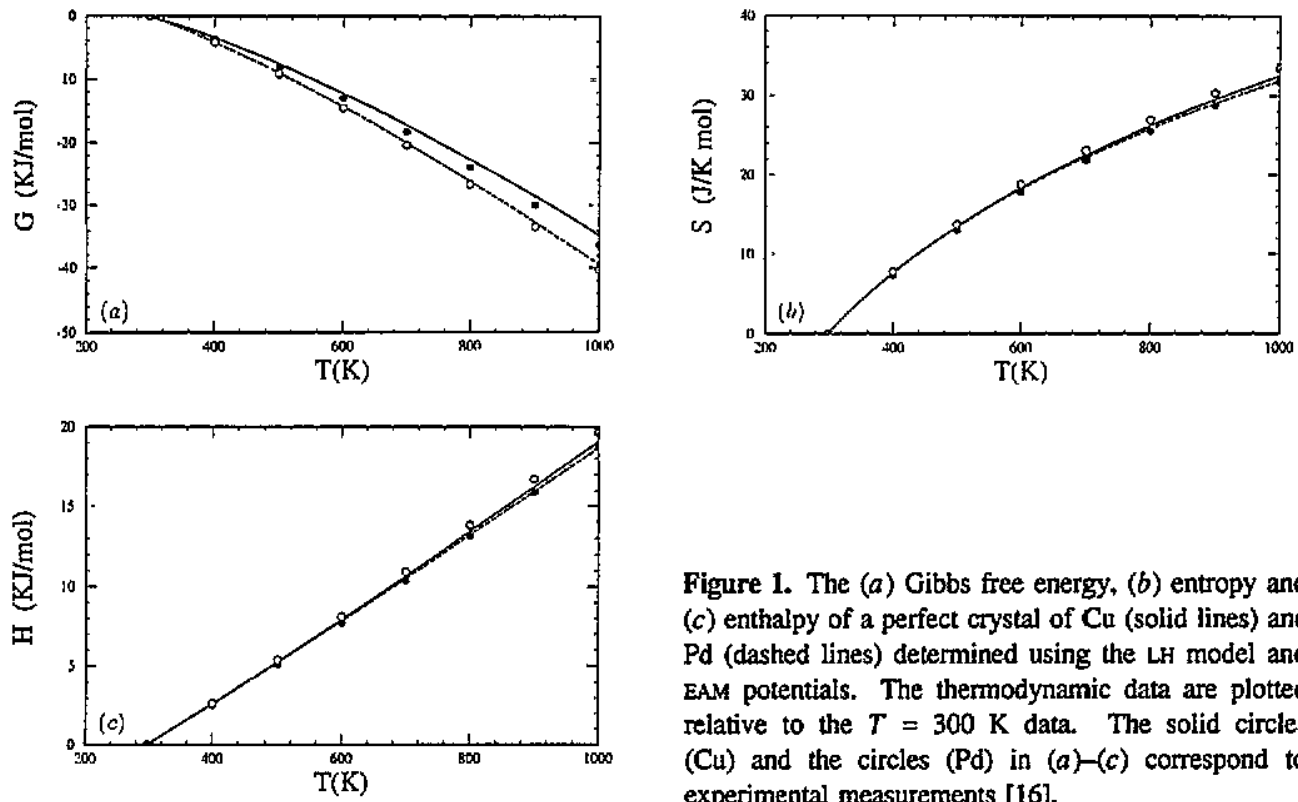

Figure 1. The (a) Gibbs free energy, (b) entropy and (c) enthalpy of a perfect crystal of $\mathrm{Cu}$ (solid lines) and Pd (dashed lines) determined using the LH model and EAM potentials. The thermodynamic data are plotted relative to the $T=300 \mathrm{~K}$ data. The solid circles $(\mathrm{Cu})$ and the circles $(\mathrm{Pd})$ in $(a)-(c)$ correspond to experimental measurements [16].

\subsection{Vacancy thermodynamics in the LH free energy minimization model}

The dependence of the relaxed Au vacancy formation free energy and volume on the number of particles in the simulation cell $N$ is indicated in figure 2 based upon $T=0 \mathrm{~K}$ simulations with $107,255,499$ and 863 particles. Since these data and similar data obtained at $600 \mathrm{~K}$ saturate rapidly with increasing simulation cell size (there is only a $0.06 \%$ and $0.05 \%$ variation in the vacancy formation free energy when the system size is increased from 255 to 863 atoms at $T=0$ and $600 \mathrm{~K}$, respectively), we choose to perform all of the remaining vacancy simulations using 255 -atom systems.

In order to expedite the simulations, we make use of the observation [18] that the free energies of the perfect crystals and many defects at finite temperature are accurately 

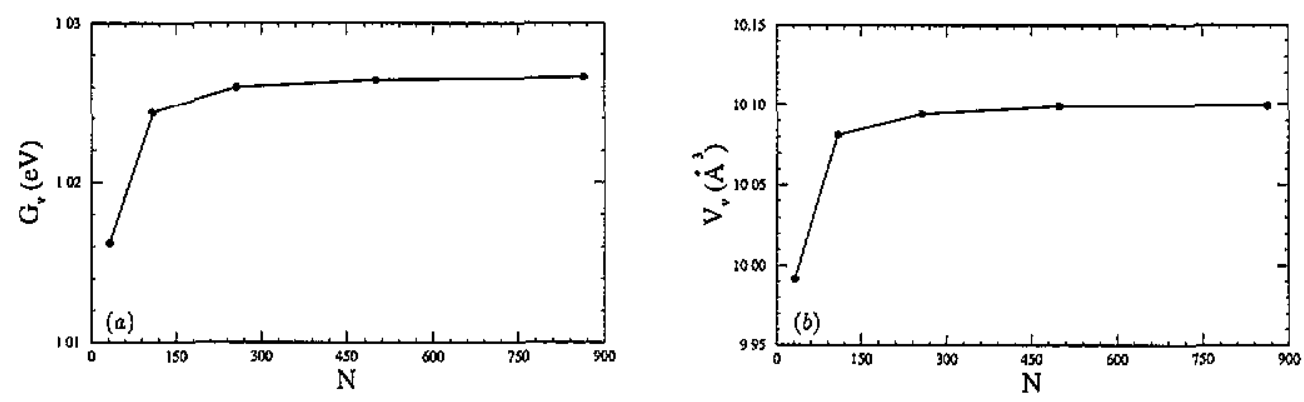

Figure 2. Sensitivity of $(a)$ the vacancy formation free energy and $(b)$ the vacancy formation volume in Au with one vacancy to variations in the number of atoms, $N$, in the simulation cell.

reproduced by determining the relaxed structure at $T=0$, rescaling the atomic coordinates in accordance with the finite temperature, perfect-crystal lattice parameter and determining the free energy as in (5). The finite temperature, perfect-crystal lattice parameter is determined within the framework of the LH free energy minimization model. Figure 3 shows the variation of the vacancy formation energy with temperature calculated using the rescaling of the relaxed, zero-temperature structure and by performing a series of LH free energy minimizations at each temperature over the same temperature range. The excellent agreement between these two curves may be attributed to the fact that aside from thermal expansion, the temperature-dependent atomic relaxations in these systems are small. In addition, small errors in the atomic positions (order $\epsilon$ ), lead to even smaller errors in the free energy (order $\epsilon^{2}$ ) due to the quadratic shape of the free energy surface near its minimum. See [18] for more details.

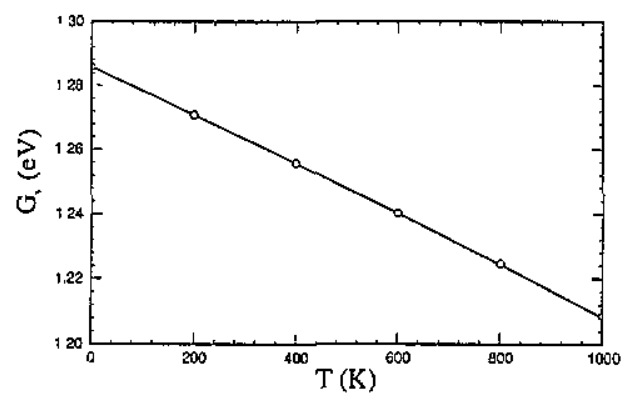

Figure 3. The vacancy formation energy for copper as a function of temperature calculated from a series of $\mathrm{uH}$ free energy minimizations (solid line-data points every $100 \mathrm{~K}$ ) and by rescaling the relaxed zero-temperature vacancy structure according to the perfect-crystal thermal expansion (open circles).

The LH determination of the vacancy formation thermodynamics (vacancy formation free energy, excess entropy, excess enthalpy and vacancy formation volume) for $\mathrm{Au}, \mathrm{Ag}, \mathrm{Cu}$, $\mathrm{Pd}, \mathrm{Pt}, \mathrm{Ni}$ are shown in figure 4 as a function of temperature. The vacancy formation free energy (figure $4(a)$ ) for all six FCC elements is a weak, nearly linear function of temperature. On average, the vacancy formation free energy varies by only $4.93 \%$ between $200 \mathrm{~K}$ and $1000 \mathrm{~K}$, with the smallest variation in $\mathrm{Au}$ and the largest variation in $\mathrm{Pt}$. While the changes in vacancy formation free energies with temperature are small, they lead to changes in the 
equilibrium vacancy concentrations of approximately $51 \%$ at $1000 \mathrm{~K}$. For example, the vacancy concentration at $1000 \mathrm{~K}$ for $\mathrm{Ag}$ is $4.1 \times 10^{-5}$ when the correct $1000 \mathrm{~K}$ vacancy formation free energy is used, while it is only $1.6 \times 10^{-5}$ based upon the formation energy determined at $200 \mathrm{~K}$. This corresponds to an error of $60 \%$. The observed small variation of the free energy with temperature leads to a small vacancy formation entropy $S_{\mathrm{v}}=-\partial G_{\mathrm{v}} / \partial T$ (figure $4(b)$ ). We find that the entropy associated with vacancy formation $\left(-\partial G_{\mathrm{v}} / \partial T\right)$ is only weakly temperature dependent at low temperatures. The vacancy formation enthalpy $\left(H_{\mathrm{v}}=G_{\mathrm{v}}+T S_{\mathrm{v}}\right)$ is nearly temperature independent (figure $4(c)$ ). The vacancy formation volume $V_{\mathrm{v}}$ (figure $4(d)$ ) also exhibits a weak temperature dependence with the values for five of the six elements within $20 \%$ of each other. $\mathrm{Ag}$, on the other hand, shows a vacancy formation volume which is nearly $50 \%$ larger than for the other FCC elements examined. In the limit of zero temperature, we have confirmed that the present vacancy formation energy and volume data agree with previously published results using the same interatomic potential [5].
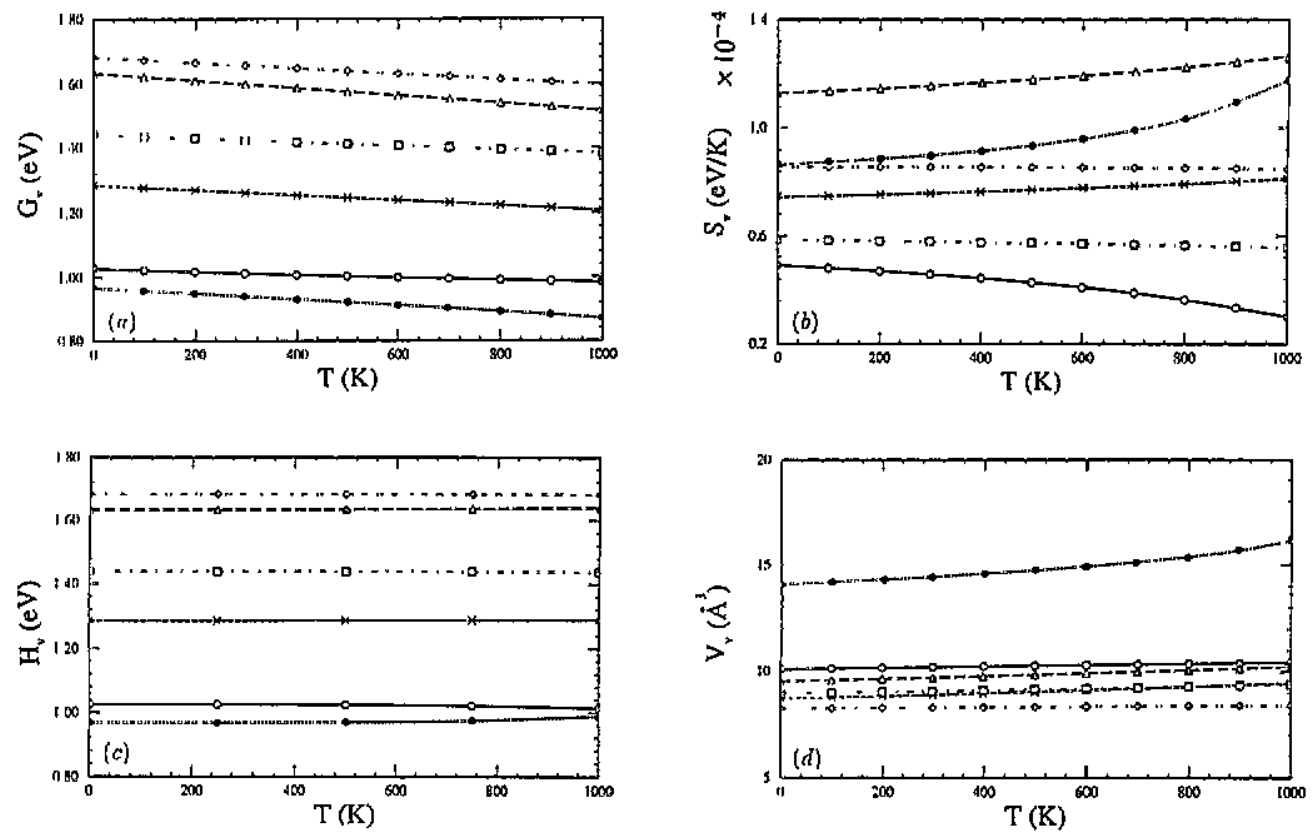

Figure 4. The vacancy formation (a) energy, (b) entropy, (c) enthalpy and $(d)$ volume plotted against temperature for an isolated vacancy in a 255-atom crystal calculated from a series of $\mathrm{LH}$ free energy minimizations for $\mathrm{Au}$ (open circle), $\mathrm{Ag}$ (solid circle), $\mathrm{Cu}$ (cross), $\mathrm{Pd}$ (square), $\mathrm{Pi}$ (dianond) and $\mathrm{Ni}$ (triangle).

The vacancy formation energy and volume are correlated with properties of the perfect crystal. For example, we note that $\mathrm{Ag}$ has the lowest sublimation energy and the lowest vacancy formation energy of the six elements examined. Pt, on the other hand, has both the largest sublimation energy and vacancy formation energy. The correlation between sublimation energy and vacancy formation energy is not completely general, however, as a few deviations are observed in the six element series examined herein. Nonetheless, such correlations are not surprising as most of the formation energy and sublimation energy are associated with the breaking of the bonds that occurs when the vacancy is created or an 
atom is removed from the crystal. A similar conelation is observed between the perfect crystal bulk modulus and largest formation volume. Ag has the smallest bulk modulus and largest formation volume, while $\mathrm{Pt}$ has the largest bulk modulus and smallest formation volume. This is not surprising as a large bulk modulus implies a small atomic relaxation when the system is perturbed by bond breaking.

\subsection{Vacancy thermodynamics in the $\mathrm{QH}$ model}

In order to test the validity of the LH model for determining vacancy formation thermodynamics, we have also performed a series of calculations within the framework of the $\mathrm{QH}$ model. Since the $\mathrm{QH}$ model explicitly includes the coupling of the vibrations of all atoms, it is more accurate than the LH model. However, because it requires the diagonalization of a $3 N \times 3 N$ matrix, it is much less computationally efficient than the $\mathrm{LH}$ model-which requires the diagonalization of $N 3 \times 3$ matrices. Because of the large overhead in determining the $\mathrm{QH}$ free energy, the finite-temperature structures used to determine the $\mathrm{QH}$ vacancy formation energies were determined using the $\mathrm{LH}$ model and hence these calculations are not self-consistent. In as much as the LH model is a reasonable approximation to the free-energy, this is a reasonable approach. However, since we are interested in determining the vacancy thermodynamics at zero pressure, we need to determine the equilibrium volume appropriate to the $\mathrm{QH}$ calculations. The equilibrium volume is determined by calculating the free energy at several lattice parameters and choosing the volume corresponding to the minimum in a parabolic fit to this data. The parabolic fits for a perfect system and a system with a vacancy in $\mathrm{Ag}$ at $800 \mathrm{~K}$ are shown in figure $5(a)$ and $(b)$, respectively.
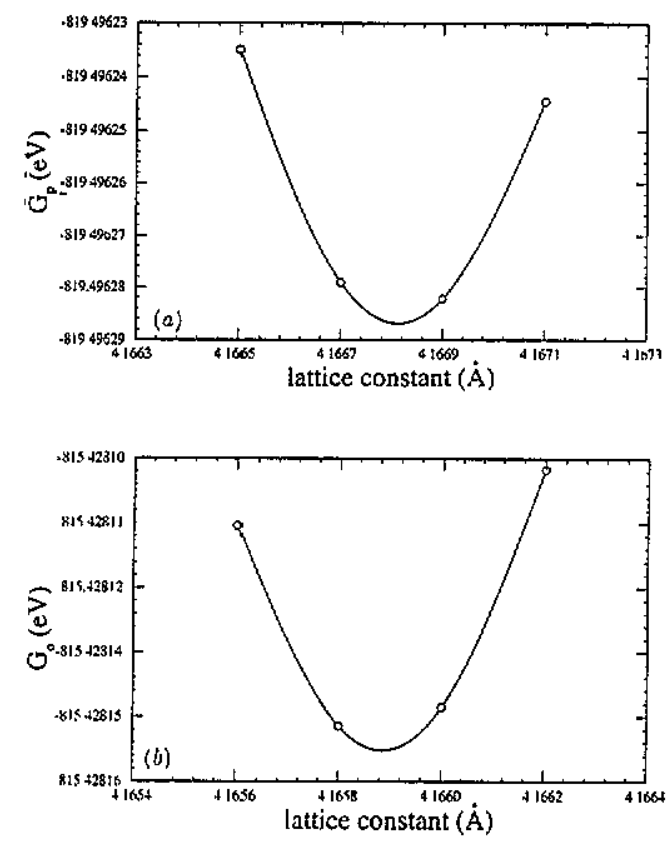

Figure 5. A parabolic fit to the $\mathrm{QH}$ Heimholtz free energy for $\mathrm{Ag}$ at $800 \mathrm{~K}$ as a function of lattice parameter $(a)$ for a perfect crystal and $(b)$ for a system with one vacancy. 
Table 1. Comparison of the vacancy formation free energy ( $G_{\mathrm{V}}$ in $\mathrm{eV}$ ) for six FCC metals using the LH method and the EAH method with third-nearest neighbours. In addition, the vacancy formation free energies obtained using the $\mathrm{QH}$ method at zero pressure are listed. The errors between the vacancy formation energies obtained in the $\mathrm{LH}$ and $\mathrm{QH}$ methods and those obtained in the ELH(3) and $\mathrm{QH}$ methods are listed as eror(1) and error(2), respectively.

\begin{tabular}{lllllll}
\hline & $T(\mathrm{~K})$ & LH & ELH & QH & $\begin{array}{l}\text { Error(1) } \\
(\%)\end{array}$ & $\begin{array}{l}\text { Error(2) } \\
(\%)\end{array}$ \\
\hline $\mathrm{Ni}$ & 200 & 1.611 & 1.606 & 1.600 & 0.7 & 0.4 \\
$\mathrm{Ni}$ & 800 & 1.542 & 1.524 & 1.503 & 5.9 & 1.4 \\
$\mathrm{Cu}$ & 200 & 1.271 & 1.264 & 1.258 & 1.0 & 0.5 \\
$\mathrm{Cu}$ & 800 & 1.227 & 1.204 & 1.181 & 3.9 & 1.9 \\
$\mathrm{Ag}$ & 200 & 0.951 & 0.946 & 0.942 & 1.0 & 0.4 \\
$\mathrm{Ag}$ & 800 & 0.896 & 0.881 & 0.867 & 3.3 & 1.6 \\
$\mathrm{Pd}$ & 200 & 1.429 & 1.422 & 1.416 & 0.9 & 0.4 \\
$\mathrm{Pd}$ & 800 & 1.397 & 1.371 & 1.350 & 3.5 & 1.6 \\
$\mathrm{Pt}$ & 200 & 1.667 & 1.658 & 1.653 & 0.8 & 0.3 \\
$\mathrm{Pt}$ & 800 & 1.618 & 1.585 & 1.566 & 3.3 & 1.2 \\
$\mathrm{Au}$ & 200 & 1.018 & 1.010 & 1.007 & 1.1 & 0.3 \\
$\mathrm{Au}$ & 800 & 0.995 & 0.969 & 0.956 & 4.1 & 1.4 \\
\hline
\end{tabular}

The free energy of vacancy formation at zero pressure for Pt calculated using both the $\mathrm{QH}$ and LH methods is plotted in figure 6 as a function of temperature. The two methods yield the same vacancy formation free energy as $T$ approaches zero and at $800 \mathrm{~K}$ the $\mathrm{LH}$ method overestimates the $\mathrm{QH}$ vacancy formation energy by only $3.3 \%$. A detailed comparison between the $\mathrm{LH}$ and $\mathrm{QH}$ determinations of the vacancy formation free energy and entropy may be made using the data in tables 1 and 2. The LH model underestimates the vacancy formation entropy over the entire temperature range $(0 \mathrm{~K}$ to $800 \mathrm{~K})$ for $\mathrm{Pt}$ by approximately $42 \%$ at $800 \mathrm{~K}$. Similar results were found for all of the elements. The excellent agreement in the free energies and the poor agreement in the entropies indicate that the vacancy formation free energy is dominated by the enthalpy and entropy associated with vacancy formation is quite small.

Table 2. Comparison of the vacancy formation entropy $S_{\mathrm{V}}$ (in $10^{-5} \mathrm{eV} \mathrm{K}^{-1}$ ) for six FCC metals using the LH method and the EAH method with third-nearest neighbours. In addition, the vacancy formation free energies using the $\mathrm{QH}$ method at zero pressure are listed. The errors between the vacancy formation energies obtained in the $\mathrm{LH}$ and QH methods and those obtained in the ELH(3) and $\mathrm{QH}$ methods are listed as error(1) and error(2), respectively.

\begin{tabular}{lrrrrll}
\hline & $T(\mathrm{~K})$ & LH & ELH(3) & QH & $\begin{array}{l}\text { Eror(1) } \\
(\%)\end{array}$ & $\begin{array}{l}\text { Error(2) } \\
(\%)\end{array}$ \\
\hline $\mathrm{Ni}$ & 200 & 11.1 & 13.5 & 16.2 & 31.5 & 16.7 \\
$\mathrm{Ni}$ & 800 & 11.9 & 13.9 & 15.6 & 23.7 & 10.9 \\
$\mathrm{Cu}$ & 200 & 7.1 & 10.0 & 13.2 & 46.2 & 24.0 \\
$\mathrm{Cu}$ & 800 & 7.5 & 10.1 & 12.5 & 40.0 & 19.5 \\
$\mathrm{Ag}$ & 200 & 8.6 & 10.7 & 12.7 & 32.3 & 15.7 \\
$\mathrm{Ag}$ & 800 & 10.1 & 11.1 & 12.3 & 17.9 & 9.8 \\
$\mathrm{Pd}$ & 200 & 5.4 & 8.7 & 11.6 & 53.4 & 25.0 \\
$\mathrm{Pd}$ & 800 & 5.2 & 8.3 & 10.5 & 50.5 & 21.0 \\
$\mathrm{Pt}$ & 200 & 8.2 & 11.6 & 15.1 & 45.7 & 23.2 \\
$\mathrm{Pt}$ & 800 & 8.1 & 11.8 & 13.9 & 41.7 & 15.1 \\
$\mathrm{Au}$ & 200 & 4.4 & 8.0 & 9.6 & 54.2 & 16.7 \\
$\mathrm{Au}$ & 800 & 3.3 & 6.4 & 7.1 & 53.5 & 9.8 \\
\hline
\end{tabular}


We note that the $\mathrm{QH}$ vacancy simulation results requires approximately a factor of over 200 more computer time to obtain than the LH model computations. This large computational advantage enjoyed by the LH method increases dramatically with increasing complexity of the defect studied.

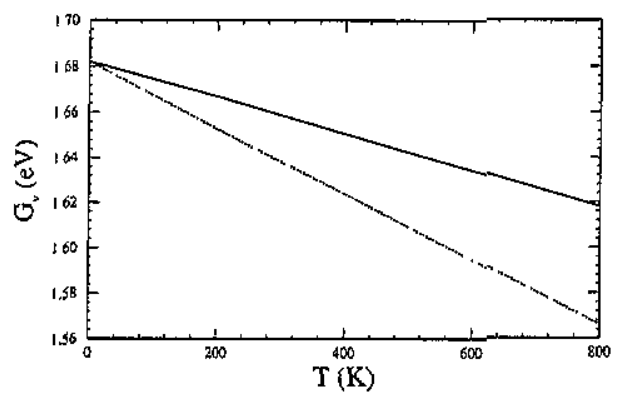

Figure 6. The vacancy formation energy versus $T$ for one vacancy in Pt. The solid curve is from the LH model, and the dotted curve from the $\mathrm{QH}$ model.

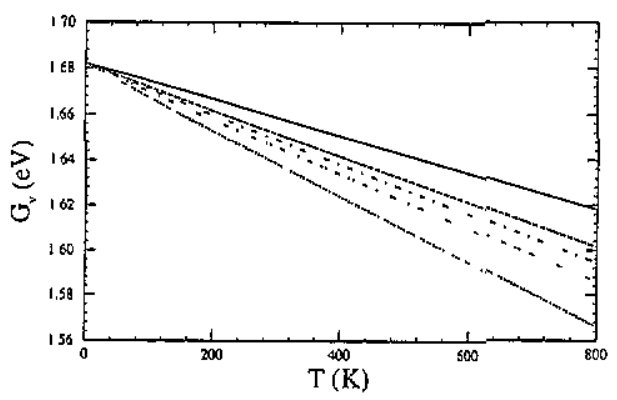

Figure 7. The vacancy formation energy as a function of temperature for Pt as determined by the extended LH model using the first-nearest neighbour correction (dashed line), the second-nearest neighbour correction (dash-dotted line), the third-nearest neighbour correction (dash-dot-dotted line). The solid curve is from the $\mathrm{LH}$ model, the dotted curve from the $\mathrm{QH}$ model,

\section{Extensions of the LH model}

The comparison between the vacancy formation entropies obtained with the LH and QH methods points out that the approximations inherent to the $\mathrm{LH}$ model are too severe to reliably predict the very small variations in vacancy formation entropy with temperature. At the same time, the computational demands of determining the free energy based upon the $\mathrm{LH}$ model are over 200 times less that for the $\mathrm{QH}$ model in the present calculations. Therefore, we have attempted to improve the accuracy of the LH model, while at the same time retaining its computational efficiency.

The main difference between the $\mathrm{LH}$ and $\mathrm{OH}$ methods is the explicit assumption that atomic vibrations are uncorrelated. These correlations are expected to be greatest between 
atoms which are near neighbours. In order to properly account for these correlations, we extend the LH model by explicitly including correlations amongst neighbouring atoms. This is accomplished by including terms in the local dynamical matrix due to all atoms out to some predetermined distance from the atom of interest. The free energy in this ELH model can be expressed as:

$$
A=U+k T \sum_{i=1}^{N} \frac{1}{n_{i}} \sum_{\beta=1}^{3 n_{i}} \ln \left[2 \sinh \left(\frac{\hbar \omega_{i \beta}}{k T}\right)\right]
$$

where $n_{i}$ is the number of atoms within some cut-off distance of atom $i$. The frequencies $\omega_{i \beta}$ in (6) are the eigenfrequencies associated with the $3 n_{i} \times 3 n_{i}$ dynamical matrix associated with atom $i$. In this way, the spatially localized vibrational interactions between nearby atoms are accounted for. In the limit that $n \rightarrow N$, this ELH method is exactly equivalent to the QH method. At the same time, as long as $n \ll N$ the ELH method is much more computationally efficient than the $\mathrm{QH}$ method.

We have performed several calculations to determine the vacancy formation free energy within the framework of the ELH model, where $n_{i}$ includes (1) all atoms within the nearest neighbour shell of atom $i$, (2) all neighbours out to the second-nearest neighbour distance and (3) out to the third-nearest neighbour distance (the cut-off of the EAM potentials). The results for $\mathrm{Pt}$ are shown in figure 7 . For the sake of comparison the original $\mathrm{LH}$ and $\mathrm{QH}$ results are reproduced in this figure. Since the ELH method yields a different free energy than does the LH method, the thermal expansion determined using the two methods are different.

Clearly, increasing the range of the ELH method from zero (the LH model) to the thirdnearest neighbour distance produces better agreement between the ELH and QH vacancy formation free energies. We find the first-nearest neighbour correction decreases the error in the vacancy formation entropy from $42 \%$ to $29 \%$, the second-nearest neighbour correction decreases it to $22 \%$ and the third-nearest neighbour correction decreases it to $15 \%$.

In tables 1 and 2 , we compare the vacancy formation free energy and vacancy formation entropy for the six FCC metals calculated using the LH method, the QH method and the ELH method using third-nearest neighbour correction, ELH(3). The LH and QH results were determined using the same LH relaxed structure with a zero-pressure QH rescaling of the volume, as described above. The $\operatorname{ELH}(3)$ results were obtained using the atomic structure determined from the free energy minimization based upon the third-neighbour ELH method. The ELH(3) method reduces the errors in the vacancy formation energy by approximately a factor of two (to within approximately $2 \%$ of the $\mathrm{QH}$ result at $800 \mathrm{~K}$ ) compared with the $\mathrm{LH}$ method. Similarly, the ELH(3) method reduces the errors in the vacancy formation entropy by greater than a factor of three compared with the LH method. In the case of the entropy, this error is reduced from an average of $38 \%$ to an average of $14 \%$ at $800 \mathrm{~K}$. Therefore, we find that the ELH method yields vacancy formation thermodynamics in good agreement with the nominally exact $\mathrm{QH}$ method, while still retaining much of the computational efficiency of the simpler LH method. The computational efficiency of the ELH method is sufficient to allow the performance of relaxations of the atomic structure and hence obtain self-consistent vacancy formation thermodynamics.

\section{Summary}

We have determined the vacancy formation thermodynamic properties (free-energy, entropy, enthalpy, volume) for six FCC metals, described by EAM potentials, as a function of 
temperature. These results were obtained within the framework of the free energy simulation method using both the local harmonic and quasiharmonic models. We find that the variation of the vacancy formation free energy with temperature is relatively small over the entire temperature range from zero to near the melting point. These small variations are, nonetheless, sufficient to produce changes in the equilibrium vacancy concentration of approximately a factor of two at $1000 \mathrm{~K}$. The agreement between the vacancy formation free energy determined using the approximate local harmonic method are in very good agreement with those obtained using the more accurate quasiharmonic method. However, the agreement between these two methods for the vacancy formation entropy is poor. These results can be reconciled by noting that the vacancy formation entropy is very small and, hence, makes a very small contribution to the vacancy formation free energy (only about $5 \%$ at $800 \mathrm{~K}$ ). We propose an extension to the local harmonic model which improves the accuracy with which the vacancy formation entropy may be determined. This extension is based upon including the correlations between vibrations of spatially nearby atoms. While this does decrease the computational efficiency of the local harmonic model, the extended local harmonic model remains very much more computationally efficient than the quasiharmonic method.

\section{Acknowledgments}

The authors gratefully acknowledge the support of the Defence Advanced Research Projects Agency through the Office of Naval Research, Grant No N00014-91-J-4019, under whose auspices this work was performed. We acknowledge helpful comments and suggestions from J M Rickman, J B Adams and A F Voter. In addition, we thank Dr R Brown for sending us a preprint of his work.

\section{References}

[1] Gillan M J 1989 J. Phys.: Condens, Matter 1689

[2] Jaccuci G, Taylor R, Tenenbaum A and Doan N 1981 J. Phys. F: Met. Phys. 11793

[3] Jaccuci $G$ and Ronchetti M 1980 Solid State Commun. 3335

[4] Squire D R and Hoover W G 1969 J. Chem. Phys. 50701

[5] Foiles S M, Baskes M I and Daw M S 1986 Phys. Rev. B 337983

[6] Balluffi R W 1991 MRS Bull. February 23

[7] Girifalco L A 1973 Statistical Physic of Materials (New York: Wiley)

[8] LeSar R, Najafabadi R and Srolovitz D J 1989 Phys. Rev. Lett. 63624

[9] Barron T H K and Klein M L 1974 Dynamical Properties of Solids vol I ed G K Horton and A A Maradudin (Amsterdam: North-Holland) pp 39t-450

[10] Philipot S R and Rickman J M 1991 J. Chem. Phys. 9415

[11] De Lorenzi G and Jaccuci G 1986 Phys. Rev. B 331993 Marchese M, Jaccuci G and Flynn C P 1988 Phil. Mag. Lett. 57245

[12] Maroudas D and Brown R 1992 MRS Symp. Proc. vol 278, Computational Methods in Materials Science at press

[13] Lutsko J F, Wolf D and Yip S 1988 J. Chem. Phys. 886525

[14] Najafabadi R, Wang H Y, Srolovitz D J and LeSar R 1992 Acta Metall. Mater. $393071-82$

[15] Najafabadi R, Srolovitz D J and LeSar R 1990 J. Mater. Res. 52663

[16] Pearson W B 1958 Handbook of Lattice Spacings and Structures of Metals and Alloys (New York: Pergamon)

[17] Foiles S M and Adams G B 1989 Phys. Rev. B 405909

[18] Rickman J M, Najafabadi R, Zhao L and Srolovitz D J 1992 J. Phys.: Condens. Matter 44923 